\title{
Quantitative proteomic analysis comparing grades ICRS1 and ICRS3 in patients with osteoarthritis
}

\author{
DONG-SIK CHAE ${ }^{1 *}$, MI EUN KIM ${ }^{2 *}$, KYUNG-YIL KANG $^{1}$, NAE YOON LEE ${ }^{3}$, \\ WOO-SUK LEE ${ }^{4}$ and JUN SIK LEE ${ }^{2}$
}

\begin{abstract}
${ }^{1}$ Department of Orthopedic Surgery, International St. Mary's Hospital, College of Medicine, Catholic Kwandong University, Incheon 22711; ${ }^{2}$ Department of Life Science, Immunology Research Lab, BK21-Four Education Research Group, College of Natural Sciences, Chosun University, Gwangju 61452;

${ }^{3}$ Department of Bionano Technology, Gachon University, Seongnam, Gyeonggi-do 13120; ${ }^{4}$ Department of Orthopedic Surgery, Yonsei University College of Medicine, Gangnam Severence Hospital, Seoul 135720, Republic of Korea
\end{abstract}

Received June 2, 2021; Accepted September 7, 2021

DOI: 10.3892/etm.2021.10905

\begin{abstract}
Osteoarthritis (OA), which is caused by joint damage, is the most common form of arthritis, affecting millions of people worldwide. This damage can accumulate over time, which is why aging is one of the main contributors to joint damage associated with OA. The OA-related proteins that have been reported to date have been identified by the comparative analysis of OA patients with normal controls, following surgical or pharmacological treatment. For the first time, the present study analyzed OA-related proteins in patients with OA according to the International Cartilage Repair Society (ICRS) scale. Changes in protein expression can be observed during the OA process. The present study demonstrated differential protein expression patterns in articular cartilage from ICRS1and ICRS3-graded OA patients. ICRS grade-matched OA knee samples from 12 OA patients, 6 ICRS grade 1 patients and 6 ICRS3 patients were subjected to proteomic analysis using the LTQ-Orbitrap mass spectrometry system. A total of 231 unique proteins were identified as expressed across the ICRS1 and ICRS3 OA patient groups. Relative differences in protein expression associated with the following classifications were observed: Biological adhesion, cell killing, cellular process,
\end{abstract}

Correspondence to: Professor Jun Sik Lee, Department of Life Science, Immunology Research Lab, BK21-Four Education Research Group, College of Natural Sciences, Chosun University, Building 3108, Pilmun-daero, Dong, Gwangju 61452, Republic of Korea

E-mail: junsiklee@chosun.ac.kr

Dr Woo-Suk Lee, Department of Orthopedic Surgery, Yonsei University College of Medicine, Gangnam Severence Hospital, 211 Eonju-ro, Seoul 135720, Republic of Korea

E-mail:wsleeos@yuhs.ac.kr

*Contributed equally

Key words: osteoarthritis, International Cartilage Repair Society, proteome analysis development process and molecular function. Although some of these proteins have been previously reported to be associated with rheumatoid arthritis, including cartilage oligomeric matrix protein, collagen types, angiogenin, complement C5 and CD59 glycoprotein, numerous additional proteins were newly identified, which may further help our understanding of disease pathogenesis. These findings suggested that these proteins may be used to develop novel therapeutic targets for $\mathrm{OA}$.

\section{Introduction}

Osteoarthritis (OA) continues to increase in prevalence, due to population aging, prolonged life expectancy and obesity. Patients with OA suffer from low quality of life associated with chronic pain and reduced joint function (1). Globally, musculoskeletal disorders represent a high proportion of conditions associated with years lived with disability, and a sharp increase in the incidence of musculoskeletal disorders was observed between 1990 and 2015 (2). OA is a representative musculoskeletal disorder in which joint function becomes irreversibly damaged due to aging, cartilage wear, trauma and sustained joint inflammation (3). Knee-joint OA can easily be diagnosed by X-ray radiography and is characterized by the narrowing of the joint space, development of osteophytes, sclerosis of the subchondral bone and cyst formation (4). According to the Kellgren-Lawrence (KL) scale, four grades of OA can be distinguished by X-ray observation (5). Throughout the various stages of osteoarthritis, exercise, medication and injection therapy have been shown to be very effective for mitigating symptoms and have been used successfully (6).

The rising prevalence of $\mathrm{OA}$ has resulted in a recent increase in the incidence of osteoporosis (7). Osteoporosis is one of the most common public health problems in modern society. Osteoporosis is characterized as a chronic skeletal disorder that features the whole-body loss of bone mineral density and micro-architectural bone tissue deterioration $(8,9)$. Patients who suffer from osteoporosis and osteoporotic fractures are vulnerable to high rates of postoperative morbidity and mortality $(10,11)$. 
Patients who suffer from OA in an aging society are eager to improve their quality of life. Thus, medical demand for OA treatments is expected to grow rapidly. Prolonged life expectancy requires appropriate treatments to ensure optimal conditions and retained knee joint function $(12,13)$. An accurate understanding of the disease state is necessary to obtain the best results when applying the various reported treatments. The concepts of precision medicine and patient-specific care are in line with expected medical trends. These approaches achieve the best results for patient-specific care, by matching the expression of specific proteins in OA cartilage and medical imaging data at various stages of KL. Previous studies have been conducted to determine the physiological and chemical properties of OA tissues, and numerous factors have been identified as associated with the status and severity of OA $(14,15)$.

Most of the previous proteomic analyses have been performed as comparative analyses of patient groups treated with different pharmacological or surgical methods. In the present study, the different stages of OA were divided according to the International Cartilage Repair Society (ICRS) grades (16) and the proteins expressed at each stage were compared. ICRS grade 1 is characterized by superficial cartilage lesions, whereas grade 2 describes lesions extending to $<50 \%$ of cartilage depth. Cartilage defects extending to $>50 \%$ of cartilage depth but not through the subchondral bone are categorized as ICRS grade 3, and cartilage that is severely damaged to the subchondral bone layer is scored as ICRS grade 4 . Grade 1 is nearly normal, whereas grades 3 and 4 are severely abnormal, and grade 4 can lead to the development of sclerosis or bony defects in the subchondral bone layer. The present study aimed to determine the proteomic differences in the OA knee between different ICRS grades. The changes in various proteins according to the ICRS grade were analyzed, which may highlight a novel way to develop patient-specific care or treatment for patients with OA.

\section{Materials and methods}

Patients and ICRS collection. The present study received Research Ethics Board approval from the International St. Mary's Hospital, Kwandong University College of Medicine (CKU-020-6). Informed consent was obtained from all patients. According to a report by the Health Insurance Review and Assessment Service of South Korea in 2019, the total number of knee arthroplasty surgeries among the ages of 70 and 79 was 58,788. Among them, the ratio males to females was 50,277:8,561. There are more however surgeries for OA in women than men with a ratio of $~ 5.9: 1$ (Health Insurance Review and Assessment Service. 2020, https://opendata. hira.or.kr/home.do). As a control group, ICRS grade 1 was selected. In the late stages of degenerative arthritis, cartilage loss progresses, and in grade 4 patients undergoing TKA, sclerosis progresses and the unique characteristics of cartilage are lost. Therefore, grade 3 , in which the unique characteristics of subchondral bone are maintained to some extent, was designated as the representative of the severe group. In the case of severe ICRS3, distal condyle bone stalk obtained during distal cutting of the femur was analyzed during TKA. The size of the specimen is about $2 \times 3 \mathrm{~cm}$, and when diagnosing the ICRS grade of the cartilage surface, the most characteristic part was used in the present study. The samples for the present study were obtained retrospectively from six patients with ICRS grade 1 and 6 patients with ICRS grade 3 . Patients were classified according to the ICRS grade and had not received any treatment prior to tissue collection (Table I). Patients with rheumatoid arthritis (RA) and autoimmune diseases were excluded from the study.

Sample preparation. ICRS1 and ICRS3 patient samples were obtained through distal compartment pieces of medial and lateral femoral condyles of the femur during total knee arthroplasty and kept on ice. Samples were homogenized in TissueLyser Bead Homogenizer (Qiagen $\mathrm{GmbH}$ ) and transferred into a sterile $1.5 \mathrm{ml}$ centrifuge tube and centrifuged at 15,800 $\mathrm{x} \mathrm{g}$ for $5 \mathrm{~min}$ at $4^{\circ} \mathrm{C}$ and supernatant was collected. Samples were immediately stored at $-80^{\circ} \mathrm{C}$ until further processing. In the present study, samples were blinded, thawed on ice and their total protein concentration was evaluated using a Pierce Coomassie total protein assay (Thermo Fisher Scientific, Inc.).

Sample preparation for proteomic analysis. For proteomic investigations, ICRS samples were first adjusted to $50 \mu \mathrm{g}$ total protein in $50 \mathrm{mM}$ ammonium bicarbonate $(\mathrm{ABC})$. Protein concentration was determined using Amicon ${ }^{\circledR}$ Ultra-0.5 centrifugal filter devices (10 kDa molecular weight cut-off; Merck KGaA) which were pre-equilibrated with $400 \mu \mathrm{l}$ of $50 \mathrm{mM}$ ABC. Samples were loaded, centrifuged at 15,800 x g for $35 \mathrm{~min}$ at $4^{\circ} \mathrm{C}$ and supernatant transferred into a new tube. Concentrates were collected and adjusted to a total volume of $100 \mu \mathrm{l}$ using $50 \mathrm{mM}$ ABC. Proteins were denatured using powdered urea to a final concentration of $8 \mathrm{M}$. Dithiothreitol was added to each sample to the final concentration of $10 \mathrm{mM}$ and samples were incubated at $37^{\circ} \mathrm{C}$ for $1 \mathrm{~h}$. Subsequently, alkylation with $50 \mathrm{mM}$ iodoacetamide was performed at room temperature in the dark for $45 \mathrm{~min}$. Samples were diluted fivefold with $50 \mathrm{mM} \mathrm{ABC}$ to prevent inhibition of trypsin activity by high concentrations of urea. Samples were digested with trypsin in a 1:40 ratio (trypsin to total protein) overnight at $37^{\circ} \mathrm{C}$, followed by acidification using dropwise addition of trifluoroacetic acid ( $\mathrm{pH}$ 2) to inhibit trypsin activity. Samples were decreased to $300 \mu \mathrm{l}$ via speed vacuum concentration and stored at $-20^{\circ} \mathrm{C}$ until capillary column liquid chromatography-tandem mass spectrometry was performed.

Mass spectrometry (MS) analysis. MS experiments were performed using LTQ-Orbitrap mass spectrometry system (Thermo Finnigan) equipped with nano-spray ionization sources. Data were acquired in data-dependent mode to simultaneously record full-scan mass and collision-induced dissociation (CID) spectra with multistage activation. For peptide mapping, the CID spectra were compared to the sequence of human KRS using Sequest (Bioworks; Thermo Electron). The MS/MS spectra were analyzed by Sorcerer 2 software v.4.0 (Sage-N Research Inc.) with Scaffold 4 Q+S as the search program for peptide/protein. The threshold was set to a minimum of one peptide identified with $90 \%$ confidence and the false discovery rate was automatically calculated based on default parameters from the software. The relative abundance of each identified protein in different samples was analyzed by QTools (Version 6.9.3; Nanoscience Instruments). 
Table I. Clinicopathological characteristics of patients with osteoarthritis included in the present study.

\begin{tabular}{lccc}
\hline & \multicolumn{2}{c}{ ICRS grade } & \\
\cline { 2 - 3 } Characteristics & I & III & P-value \\
\hline Age, years & $71.8 \pm 5.0$ & $74.8 \pm 3$ & 0.255 \\
Sex, W:M & $6: 0$ & $5: 1$ & 0.363 \\
BMI, $\mathrm{kg} / \mathrm{m}^{2}$ & $27.3 \pm 3.7$ & $25.5 \pm 4.0$ & 0.432 \\
K-L grade & $3.7 \pm 0.5$ & $3.2 \pm 0.4$ & 0.094
\end{tabular}

ICRS, International Cartilage Regeneration \& Joint Preservation Society; BMI, Body Mass Index; K-L grade, Kellgren-Lawrence grade; $\mathrm{M}$, men; W, women.

Bioinformatics analyses. Pathway analysis of up-/down-regulated proteins identified by LC-MS/MS was conducted using the Gene ontology (GO) annotation. GO annotation data for a variety of information is available from the UniProt-Human Protein Reference Database (http://www.hprd.org) and the Gene Ontology Consortium website. GO-based analysis was performed to classify proteins based on their biological process, molecular function, cell process and developmental process using Sorcerer 2.

Statistical analyses. Two tailed unpaired Student's t-test was used to compare patients with ICRS1 grade and patients with ICRS3 grade. $\mathrm{P}<0.05$ was considered to indicate a statistically significant difference.

\section{Results}

Holistic protein and peptide mining. Only proteins showing at least one peptide with an individual score confidence $>20$ PEAKS when the scaffold parameter was set at a protein threshold of $90 \%$ were considered as valid candidates. A total of 224 unique proteins were identified across ICRS1 and ICRS3 patient proteomic samples. When assessing each cohort individually, 198 unique proteins were identified in ICRS1 patient samples and 162 unique proteins were identified in ICRS3 patient samples. A review of the overlap between the proteomes of each cohort revealed the 220 proteins that were common to both patient groups (Fig. 1).

GO-based classification of proteins. For the following analyses, we focused on a list of reliable proteins classified into GO-based categories, including biological process (Fig. 2A), cellular component (Fig. 2B) and molecular function (Fig. 2C), according to the Human Protein Reference Database. To obtain deeper biological insights into the proteins identified in the two grade groups of OA tissues, we classified the identified proteins into GO-based categories, including biological adhesion, cell killing, cellular process, developmental process and molecular process (Fig. 2A). Proteins were mostly categorized as either involved in cellular processes $(n=142)$ or biological regulation $(\mathrm{n}=128)$ and were primarily involved in a cascade of functions, including cell growth, cell killing, adhesion, cellular process and development, and molecular functions. From the

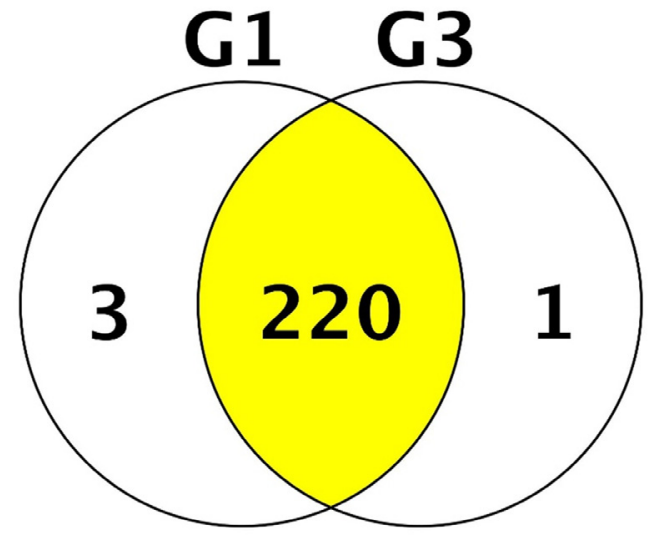

Figure 1. Venn diagram of proteins identified in ICRS patient samples. The total number of proteins identified was 234 , with three proteins detected in the ICRS1 group, one protein detected in the ICRS3 group and 230 proteins detected in both groups. ICRS, International Cartilage Repair Society.

category of proteins involved in biological processes, we selected those that showed significant log-fold differences in expression between the ICRS1 and ICRS3 groups.

Biological adhesion proteins. The first proteomic analysis was limited to the top twenty identified adhesion-related proteins. Cell adhesion proteins represent a subset of proteins that are located on the cell surface and function in cell-cell adhesion and the adhesion of cells to their surroundings, which are crucial for the maintenance of tissue structure and function (17). Cartilage oligomeric matrix protein (COMP), fibrinogen, actin, thrombospondin-4, vitronectin and lactadherin were identified as differentially expressed proteins in samples from patients with OA. COMP has recently been suggested to act as a prognostic and diagnostic marker for RA (18). Although COMP appears to be upregulated in OA, no significant difference was observed in COMP levels between ICRS1 and ICRS3 (log 0.38-fold) tissues (Table II). Notably, a 21-fold increase in thrombospondin-1 levels was observed in the ICRS3 group. Additional proteins that exhibited differential expression included various collagen (COL) proteins, including COL6A2, COL6A3 and COL14A1, showing slightly lower expression in the ICRS3 group than in the ICRS1 group.

Cell death (cell killing proteins). Cell-killing mechanisms induce cell death, resulting in the loss of biological cell functions $(19,20)$. Our proteomic analysis identified seven cell death-related proteins that showed differential expression in the ICRS3 group compared with the ICRS1 group. These proteins were the following: Lysozyme C (LYZ; -0.74-fold), peroxiredoxin-1 (PRDX1; -0.32-fold), tubulin $\beta$ chain (TUBB; - $\log 0.74$-fold), histidine-rich glycoprotein (HRG; 0.38-fold), histone H2B type 1-J (HIST1H2BJ; 1.77-fold) and serum albumin (ALB; log 21-fold; Table III). Although most of the identified proteins showed small changes, HIST1H2BJ showed a significant increase in the ICRS3 group compared with the ICRS1 group. Phosphorylated histone H2B is thought to induce cell death in joints. Although ALB has been shown to protect against cell death (21), the high ALB levels observed in the ICRS3 group suggested that this protein may be involved in the regulation of joint environment homeostasis. 
Table II. Fold change ratios of selected upregulated or downregulated protein candidates for biological adhesion proteins in ICRS1 and ICRS3 groups.

\begin{tabular}{|c|c|c|c|c|}
\hline Regulation & Protein & Gene name & Fold change (Log) & P-value \\
\hline \multirow[t]{8}{*}{ Up } & Thrombospondin-1 & THBS 1 & 21.00 & 0.28 \\
\hline & Hemoglobin subunit $\beta$ & HBB & 1.58 & 0.2 \\
\hline & Isoform $2 \mathrm{C} 2 \mathrm{~A}$ of collagen $\alpha-2$ (VI) chain & COL6A2 & 1.38 & 0.15 \\
\hline & Thrombospondin-4 & THBS4 & 1.14 & 0.13 \\
\hline & Isoform 2 of cadherin-23 & $\mathrm{CDH} 23$ & 1.00 & 0.12 \\
\hline & Cartilage oligomeric matrix protein & COMP & 0.68 & 0.32 \\
\hline & Transforming growth factor- $\beta$-induced proteinig-h3 & TGFBI & 0.38 & 0.6 \\
\hline & Apolipoprotein A-IV & APOA4 & 0.14 & 0.89 \\
\hline \multirow[t]{12}{*}{ Down } & Fibronectin & FN1 & -0.32 & 0.34 \\
\hline & Isoform 2 of collagen $\alpha-3$ (VI) chain & COL6A3 & -0.51 & 0.69 \\
\hline & Protein AMBP & AMBP & -0.51 & 0.69 \\
\hline & Isoform 2 of collagen $\alpha-1$ (XIV) chain & COL14A1 & -0.51 & 0.75 \\
\hline & Chloride intracellular channel protein 1 & CLIC1 & -0.51 & 0.71 \\
\hline & Isoform $\gamma$-A of fibrinogen $\gamma$ chain & FGG & -0.74 & 0.57 \\
\hline & Vitronectin & VTN & -0.74 & 0.18 \\
\hline & Lactadherin & MFGE8 & -0.74 & 0.49 \\
\hline & Fibrinogen $\beta$ chain & FGB & -1.32 & 0.43 \\
\hline & Actin, cytoplasmic 1 & ACTB & -1.32 & 0.13 \\
\hline & Fibrinogen $\alpha$ chain & FGA & -1.74 & 0.34 \\
\hline & $\begin{array}{l}\text { Isoform } 2 \text { of EGF-like repeat and discoidin I-like } \\
\text { domain-containing protein } 3\end{array}$ & EDIL3 & -2.32 & 0.22 \\
\hline
\end{tabular}

Up, upregulated; down, downregulated.

Cellular process proteins. A total of 107 proteins were identified that were classified as cellular process-related proteins. Cellular processes are mediated through the complex actions of several biological molecules that communicate via biophysical or biochemical interactions (22). Proteins associated with several cellular processes were identified, which presented either increased or decreased expression levels in the ICRS3 group, and changes in these protein levels can either accelerate OA progression or alleviate OA symptoms. The results from our analysis (Table IV) demonstrated that the expression levels of phosphatidylethanolamine-binding protein 1 (PEBP1; - log 1.32-fold), pigment epithelium-derived factor (SERPINF1; log 1-fold), tetranectin (CLEC3B; - $\log 2.32$-fold), apolipoprotein E (APOE; - $\log$ 2.32-fold), procollagen C-endopeptidase enhancer 1 (PCOLCE; -log 2.32-fold), collagen type I a 1 chain (COL1A1; - $\log 4.64$-fold), cartilage intermediate layer protein (CILP; -log 18.35-fold), cartilage acidic protein 1 (CRTAC1; - $\log 19.93$-fold) and collagen type I $\alpha 2$ chain (COL1A2; - $\log 23.25$-fold) were decreased in the ICRS3 group compared with the ICRS1 group. Conversely, the expression levels of apolipoprotein B-100 (APOB; log 2.04-fold), isoform 2C2A of the collagen type VI $\alpha 2$ chain (COL6A2; log 1.38-fold), antithrombin-III (SERPINC; log 1.2-fold) and prelamin-A/C (LMNA; log 17.82-fold) were increased in the ICRS3 group compared with the ICRS1 group. The results confirmed that the expression level of LMNA was remarkably high in the ICRS3 group, and that LMNA high expression level and the presence of LMNA mutations are known to be contributors of the apoptosis of OA chondrocytes $(23,24)$.

Developmental process proteins. The results from proteomic assay identified 51 developmental process-related proteins. Proteins involved in the developmental process, which describe the processes through which multicellular organisms develop from the early immature stage, are responsible for the maturity and differentiation of various organisms (25-27). Of the 51 identified proteins, the following proteins showed significantly decreased expression levels in the ICRS3 group compared with the ICRS1 group: Glutathione 5-transferase P (G5TP1; -log 1.00-fold), chondroadherin (CHAD; - $\log 1.32$-fold), angiogenin (ANG; -log 1.74-fold), procollagen C-endopeptidase enhancer 1 (PCOLCE; - log 2.32-fold), tetranectin (CLEC3B; - -log 2.32-fold) and collagen type I $\alpha 1$ chain (COL1A1; - $\log$ 4.64-fold). Conversely, the expression levels of apolipoprotein A-II (APOA2; log 1.32-fold) and collagen type $\mathrm{V} \alpha 2$ chain (COL5A2; log 8.57-fold) were significantly increased in the ICRS3 group compared with the ICRS1 group (Table V).

Growth and molecular function proteins. Growth and molecular function-related proteins not only play important roles in cell and tissue growth but are also important for cartilage regeneration related to OA $(28,29)$ In the present study, we identified 4 and 114 proteins related to growth and molecular functions, respectively, and we confirmed that their expression levels 
A

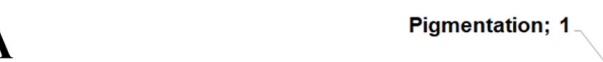

\section{Growth; 4}

Rhythmic process; 4

Unknown; 119

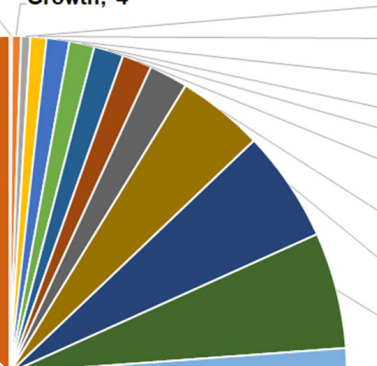

Cell killing; 7

Viral process; 10

Locomotion; 11

Reproduction; 13

Reproductive process; 13

Biological adhesion; 17

Multi-organism process; 38

Immune system process; 49

Developmental process; 51

Biological regulation; 97
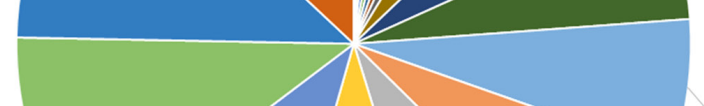

Establishment of localization; 60

Localization; 64

Response to stimulus; 94
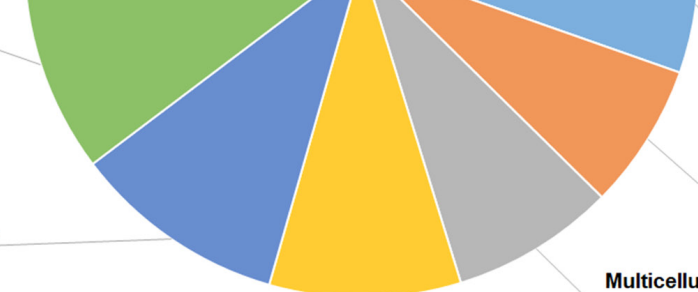

Multicellular organismal process; 72

Metabolic process; 84

B

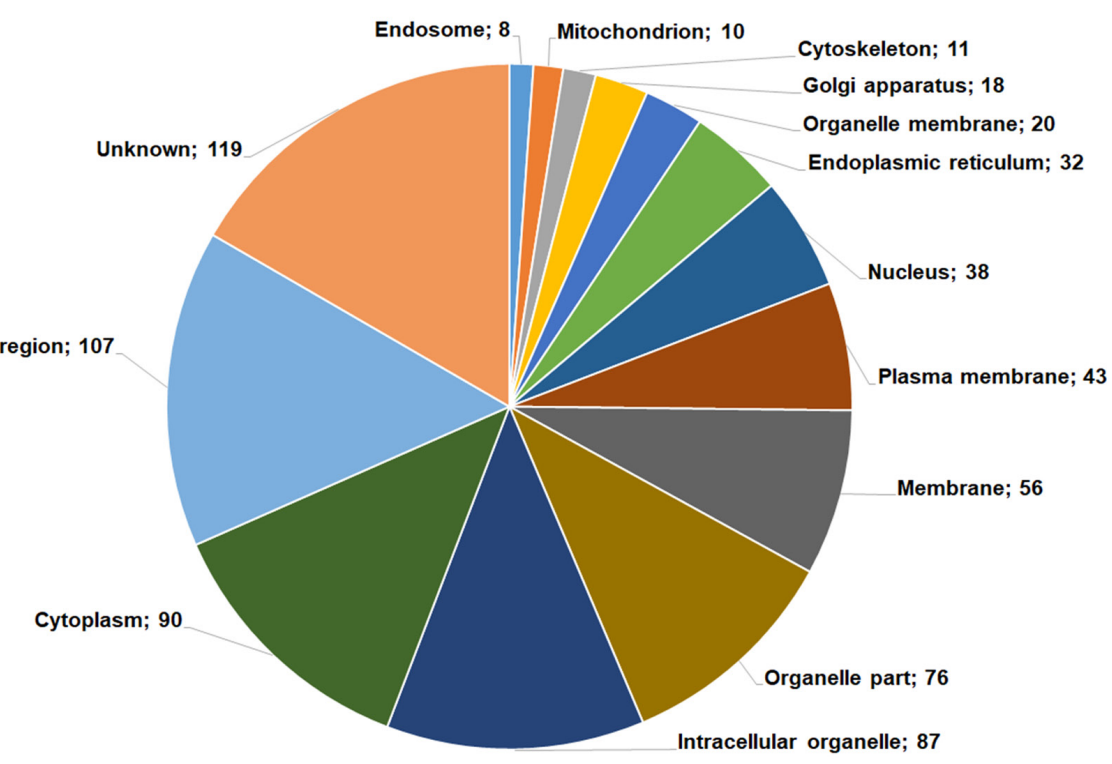

C

Chemoattractant activity; $1 \quad$ Motor activity; 1 Chemorepellent activity; 1

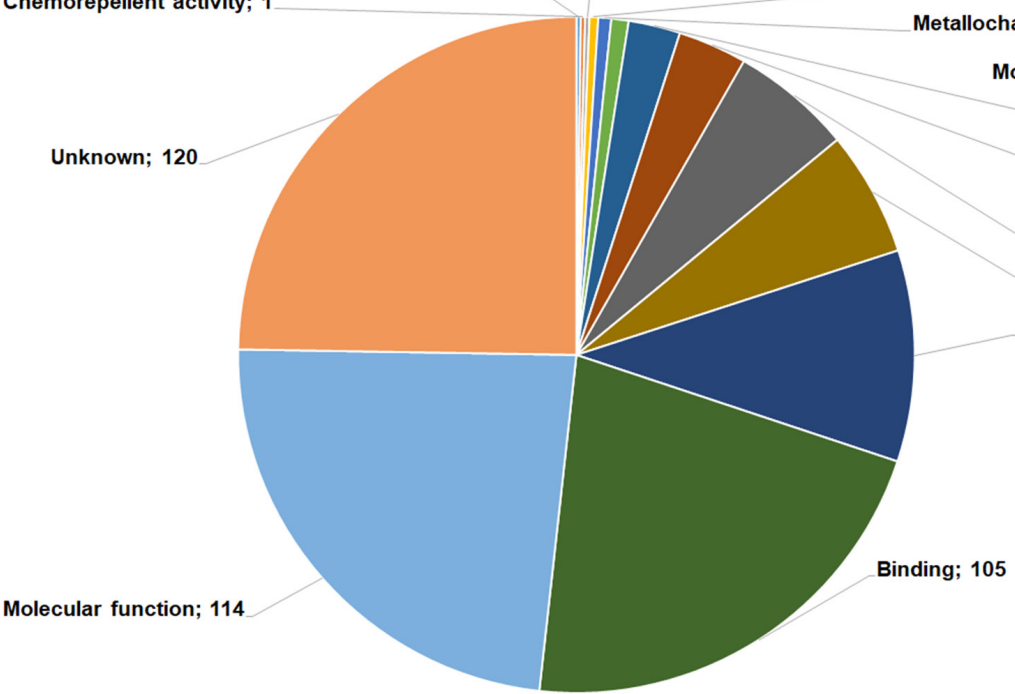

Electron carrier activity; 2

etallochaperone activity; 3

Molecular transducer activity; 4

Antioxidant activity; 12

Transporter activity; 16

Structural molecule activity; 28

Enzyme regulator activity; 29

Catalytic activity; 49

Figure 2. Pie chart representations of proteins according to (A) biological process, (B) cellular component and (C) molecular function. 
Table III. Fold-change ratios of selected upregulated or downregulated protein candidates for cell killing proteins in ICRS1 and ICRS3 groups.

\begin{tabular}{lllcc}
\hline Regulation & \multicolumn{1}{c}{ Protein } & Gene name & Fold change (Log) & P-value \\
\hline Up & Serum albumin & ALB & 21.00 & 0.88 \\
& Histone H2B type 1-J & HIST1H2BJ & 1.77 & 0.44 \\
& Glyceraldehyde-3-phosphate dehydrogenase & GAPDH & 0.38 & 0.23 \\
\multirow{3}{*}{ Down } & Histidine-rich glycoprotein & HRG & 0.38 & 0.8 \\
& Peroxiredoxin-1 & PRDX1 & -0.32 & 0.77 \\
& Lysozyme C & LYZ & -0.74 & 0.66 \\
& Tubulin $\beta$ chain & TUBB & -0.74 & 0.42 \\
\hline
\end{tabular}

Up, upregulated; down, downregulated.

Table IV. Fold-change ratios of selected up-regulated or down-regulated protein candidates for cellular process proteins in ICRS1 and ICRS3 groups.

\begin{tabular}{|c|c|c|c|c|}
\hline Regulation & Protein & Gene name & Fold-change (Log) & P-value \\
\hline \multirow[t]{12}{*}{ Up } & Thrombospondin-1 & THBS1 & 21.00 & 0.28 \\
\hline & Prelamin-A/C & LMNA & 17.81 & 0.34 \\
\hline & Apolipoprotein B-100 & APOB & 2.04 & 0.14 \\
\hline & Isoform $2 \mathrm{C} 2 \mathrm{~A}$ of Collagen $\alpha-2(\mathrm{VI})$ chain & COL6A2 & 1.38 & 0.15 \\
\hline & Hemoglobin subunit $\delta$ & HBD & 1.32 & 0.46 \\
\hline & Fibulin-1 & FBLN1 & 1.20 & 0.32 \\
\hline & Antithrombin-III & SERPINC1 & 1.20 & 0.17 \\
\hline & Thrombospondin-4 & THBS4 & 1.14 & 0.13 \\
\hline & Carbonic anhydrase 1 & CA1 & 1.14 & 0.36 \\
\hline & Superoxide dismutase $[\mathrm{Cu}-\mathrm{Zn}]$ & SOD1 & 1.07 & 0.2 \\
\hline & Isoform 2 of cadherin- 23 & $\mathrm{CDH} 23$ & 1.00 & 0.13 \\
\hline & Retinol-binding protein 4 & RBP4 & 1.00 & 0.017 \\
\hline \multirow[t]{18}{*}{ Down } & $\alpha$-1-antitrypsin & SERPINA1 & -1.00 & 0.15 \\
\hline & Lumican & LUM & -1.00 & 0.12 \\
\hline & Pigment epithelium-derived factor & SERPINF1 & -1.00 & 0.36 \\
\hline & Fibrinogen $\beta$ chain & FGB & -1.32 & 0.43 \\
\hline & Actin, cytoplasmic 1 & АСТВ & -1.32 & 0.13 \\
\hline & Biglycan & BGN & -1.32 & 0.023 \\
\hline & $\alpha$-1-antichymotrypsin & SERPINA3 & -1.32 & 0.27 \\
\hline & Phosphatidylethanolamine-binding protein 1 & PEBP1 & -1.32 & 0.067 \\
\hline & Argininosuccinate synthase & ASS1 & -1.32 & 0.48 \\
\hline & Fibrinogen $\alpha$ chain & FGA & -1.74 & 0.34 \\
\hline & Complement component $\mathrm{C} 8 \alpha$ chain & $\mathrm{C} 8 \mathrm{~A}$ & -1.74 & 0.27 \\
\hline & Apolipoprotein E & APOE & -2.32 & 0.24 \\
\hline & Procollagen C-endopeptidase enhancer 1 & PCOLCE & -2.32 & 0.081 \\
\hline & Tetranectin & CLEC3B & -2.32 & 0.073 \\
\hline & Collagen $\alpha-1$ (I) chain & COL1A1 & -4.64 & 0.35 \\
\hline & Cartilage intermediate layer protein 1 & CILP & -18.35 & 0.59 \\
\hline & Cartilage acidic protein 1 & CRTAC1 & -19.93 & 0.15 \\
\hline & Collagen $\alpha-2$ (I) chain & COL1A2 & -23.25 & 0.34 \\
\hline
\end{tabular}

Up, upregulated; down, downregulated.

were increased or decreased in the ICRS3 group compared with the ICRS1 group. Metallothionein-2 (MT2A; - $\log 1.00$-fold), metallothionein-1X (MT1X; -log 1.32-fold), argininosuccinate synthase (ASS1; - $\log 1.32$-fold), isoform 2 of N-acetlymuramoyl-L-alanine amidase (PGLYRP2; -log 1.74-fold) and CD59 glycoprotein (CD59; -log 1.74-fold) 
Table V. Fold-change ratios of selected up-regulated or down-regulated protein candidates for developmental process proteins in ICRS1 and ICRS3 groups.

\begin{tabular}{|c|c|c|c|c|}
\hline Regulation & Protein & Gene name & Fold-change (Log) & P-value \\
\hline \multirow[t]{5}{*}{ Up } & Collagen $\alpha-2(\mathrm{~V})$ chain & COL5A2 & 8.57 & 0.34 \\
\hline & Apolipoprotein A-II & APOA2 & 1.32 & 0.33 \\
\hline & Fibulin-1 & FBLN1 & 1.20 & 0.32 \\
\hline & Isoform 2 of cadherin- 23 & $\mathrm{CDH} 23$ & 1.00 & 0.10 \\
\hline & Annexin A2 & ANXA2 & 1.00 & 0.48 \\
\hline \multirow[t]{9}{*}{ Down } & Glutathione S-transferase P & GSTP1 & -1.00 & 0.19 \\
\hline & Actin, cytoplasmic 1 & ACTB & -1.32 & 0.13 \\
\hline & Phosphatidylethanolamine-binding protein 1 & PEBP1 & -1.32 & 0.067 \\
\hline & Chondroadherin & CHAD & -1.32 & 0.42 \\
\hline & Argininosuccinate synthase & ASS1 & -1.32 & 0.48 \\
\hline & Angiogenin & ANG & -1.74 & 0.21 \\
\hline & Procollagen C-endopeptidase enhancer 1 & PCOLCE & -2.32 & 0.081 \\
\hline & Tetranectin & CLEC3B & -2.32 & 0.073 \\
\hline & Collagen $\alpha-1$ (I) chain & COL1A1 & -4.64 & 0.35 \\
\hline
\end{tabular}

Up, upregulated; down, downregulated.

Table VI. Fold-change ratios of selected upregulated or downregulated protein candidates for growth and molecular function process proteins in ICRS1 and ICRS3 groups.

\begin{tabular}{|c|c|c|c|c|}
\hline Regulation & Protein & Gene name & Fold-change (Log) & P-value \\
\hline \multirow[t]{10}{*}{ Up } & Complement C5 & C5 & 19.8 & 0.13 \\
\hline & Ryanodine receptor 1 & RYR1 & 18.38 & 0.34 \\
\hline & Apolipoprotein E & APOE & 2.32 & 0.24 \\
\hline & Apolipoprotein B-100 & APOB & 2.04 & 0.14 \\
\hline & Peroxiredoxin-2 & PRDX2 & 1.63 & 0.15 \\
\hline & Carbonic anhydrase 1 & CA1 & 1.14 & 0.36 \\
\hline & Thrombospondin-4 & THBS4 & 1.14 & 0.13 \\
\hline & Lumican & LUM & 1 & 0.12 \\
\hline & Complement factor $\mathrm{H}$-related protein 1 & CFHR1 & 1.00 & 0.57 \\
\hline & Profilin-1 & PFN1 & 0.32 & 0.76 \\
\hline \multirow[t]{10}{*}{ Down } & Pigment epithelium-derived factor & SERPINF1 & -1.00 & 0.36 \\
\hline & Metallothionein-2 & MT2A & -1.00 & 0.58 \\
\hline & $\alpha-1$-antichymotrypsin & SERPINA3 & -1.32 & 0.27 \\
\hline & Phosphatidylethanolamine-binding protein 1 & PEBP1 & -1.32 & 0.067 \\
\hline & Metallothionein-1X & MT1X & -1.32 & 0.5 \\
\hline & Argininosuccinate synthase & ASS1 & -1.32 & 0.48 \\
\hline & Isoform 2 of $\mathrm{N}$-acetylmuramoyl-L-alanine amidase & PGLYRP2 & -1.74 & 0.39 \\
\hline & Annexin A1 & ANXA1 & -1.74 & 0.062 \\
\hline & Complement component $\mathrm{C} 8 \alpha$ chain & $\mathrm{C} 8 \mathrm{~A}$ & -1.74 & 0.27 \\
\hline & CD59 glycoprotein & CD59 & -1.74 & 0.36 \\
\hline
\end{tabular}

Up, upregulated; down, downregulated.

expression levels were decreased in the ICRS3 group compared with the ICRS1 group. Conversely, the expression levels of apolipoprotein B-100 (APOB; log 2.04-fold), ryanodine receptor (RYR1; log 18.38-fold) and complement C5 (C5; log 19.8-fold) were increased in the ICRS3 group compared with the ICRS1 group. Interestingly, the expression level of C5 has been reported to be increase in RA (30). In the present study, the identification of upregulated C5 expression in OA suggested that increased expression of C5 may serve as a major factor in OA development (Table VI). 


\section{Discussion}

In the present study, we determined the differentially expressed proteins between ISCR 1 and ISCR 3 grade OA patients and analyzed these proteins according to the GO-based classification of protein function. Most previous reports have conducted proteomic analyses of OA patients compared with control groups, following pharmacological or surgical treatments $(31,32)$. In previous studies, many proteomic analyses for arthritis have been reported. However, most studies did not accurately differentiate between RA and degenerative arthritis. Furthermore, a number of biomarkers for RA are involved in inflammatory diseases and autoimmune diseases (33-36). The present study aimed to determine biomarkers in OA, which differs from rheumatism, and performed proteomic analysis according to ICRS grade. The present study analyzed the differential expression of proteins in tissue samples from OA patients with early or late ICRS stages, in order to identify potential OA-inducing factors. Our protein analysis identified numerous proteins, some of which may be considered as potential parameters associated with the progression of OA.

Following analysis of the proteins characterized as biological adhesion proteins, COMP was found to be highly expressed in OA patients; however, there was no significant difference in COMP expression levels between the ICRS1 and ICRS3 grades. This result suggested that COMP may act as an OA-inducing factor but may not affect ICRS grade. Interestingly, thrombospondin-1 expression was increased 21-fold in the ICRS3 group compared with the ICRS1 group. Thrombospondin-1 can bind to fibrinogen, fibronectin, laminin and type V collagen (37) and has been shown to play certain roles in platelet aggregation, tumorigenesis and angiogenesis (38-42). Both the procollagen domain and the type I repeats of thrombospondin-1 have been reported to inhibit neovascularization and endothelial cell migration. Thrombospondin-1 contains three type I repeats, although only the last two have been found to inhibit angiogenesis $(43,44)$. Thrombospondin-1 has also been shown to interact with matrix metalloproteinase 2 (45). Thrombospondin-1 was found to be highly expressed in $\mathrm{OA}$ and may regulate inflammation in the joint area $(46,47)$. Furthermore, the expression levels of COL1A1 (-log 4.64-fold) and COL1A2 (-log 23.25-fold) were significantly decreased in the ICRS3 group compared with the ICRS1 group in the present study. Increased COL1A1 and COL1A2 expression levels have been reported to reduce OA progression (48). In the present study, the decreased expression levels of COL1A1 and COL1A2 in the ICRS3 group compared with the ICRS1 group suggested that changes in their expression may be associated with OA progression.

Angiogenin levels in the synovial fluid have been reported to be increased in patients with inflammatory diseases compared with healthy individuals (49). Furthermore, highest angiogenin levels in the synovial fluid have been reported for patients with active RA or acute gout (49). However, the present study demonstrated that angiogenin expression was higher in the ICRS1 group than in the ICRS3 group. Tetranectin is a phosphorylated glycoprotein that has been hypothesized to regulate mineral deposition within the bone $(50,51)$; however, the role of tetranectin in the pathogenesis of OA is currently unclear. Tetranectin has been implicated in the impaired regulation of fibrinolysis and is associated with the inflammatory process in RA $(52,53)$. However, the present study demonstrated that tetranectin expression was decreased in the ICRS3 group compared with the ICRS1 group.

The presents study demonstrated that the expression levels of C5 and RYR1 were significantly increased in the ICRS3 group compared with the ICRS1 group. Interestingly, C5 expression was upregulated 19.8-fold in the ICRS3 group compared with the ICRS1 group. The cleavage of C5 into C5a and C5b results in the formation of the C5b-9 complex through the recruitment of downstream complement proteins. The C5a receptor is expressed in the cartilage of both normal subjects and patients with OA and RA, and C5 receptor expression is induced by interleukin-1 $\beta$ in chondrocytes (54-56). C5 activation in OA may affect the experience of pain, which is a specific clinical feature of OA, as C5 plays a vital role in the onset of pain via the activation and sensitization of nociceptors, both in vitro and in vivo (57). In addition, antagonists or inhibitors of C5a receptors might represent key candidates for pain therapy in OA. As OA progresses and the ICRS grade increases, the expression of C5 also appears to be increased, which may cause patients to feel increasing pain. In addition, the membrane attack complex (MAC) inhibitory protein CD59 is likely involved in the protection against OA (57). Consistent with this finding, the present study demonstrated that CD59 expression was decreased in the ICRS3 group compared with the ICRS1 group, suggesting that OA progression may be further accelerated by MAC activity.

In summary, the present comprehensive analysis of protein expression differences between ICRS grades demonstrated that most OA-associated protein expression levels were increased during the early ICRS stages and decreased as the ICRS grade progresses. Furthermore, protein analysis on bone tissues samples was performed according to ICRS grade. Comparative analysis of proteins in bone tissues and blood will be performed in future experiments. Collectively, the findings of the present study suggested that the protein expression patterns associated with OA onset and those associated with ICRS progression may differ. Moreover, results of the present study suggested a potential novel treatment option for the target of thrombospondin-1 and C5, which were expressed in ICRS3 stage.

\section{Acknowledgements}

Not applicable.

\section{Funding}

This study was supported by the Ministry of Health \& Welfare (grant no. HI18C0661), Republic of Korea.

\section{Availability of data and materials}

The datasets used and/or analyzed during the current study are available from the corresponding author on reasonable request.

\section{Authors' contributions}

DSC, MEK, WSL and JSL conceived and designed the experiments. MEK, DSC, KYK and NYL performed experiments 
and analyzed the data. DSC and JSL wrote the paper. DSC and MEK confirm the authenticity of all the raw data. All authors have read and approved the final manuscript.

\section{Ethics approval and consent to participate}

This study was approved by the Research Ethics Board from the International St. Mary's Hospital, Kwandong University College of Medicine (approval no. CKU-020-6). Written consent was obtained from all patients.

\section{Patient consent for publication}

Not applicable.

\section{Competing interests}

A patent was submitted for the diagnostic prognosis kit used in the present study (www.kipris.or.kr; title, Biomarkers for osteoarthritis and osteoarthritis diagnostic or prognostic kit; registration no, 101848064; approval date, 04/05/2018; inventor, Jun Sik Lee).

\section{References}

1. Martel-Pelletier J, Barr AJ, Cicuttini FM, Conaghan PG, Cooper C, Goldring MB, Goldring SR, Jones G, Teichtahl AJ and Pelletier JP: Osteoarthritis. Nat Rev Dis Primers 2: 16072, 2016.

2. Disease GBD, Injury I and Prevalence C; GBD 2015 Disease and Injury Incidence and Prevalence Collaborators: Global, regional, and national incidence, prevalence, and years lived with disability for 310 diseases and injuries, 1990-2015: A systematic analysis for the Global Burden of Disease Study 2015 Lancet 388: 1545-1602, 2016.

3. Sokolove J and Lepus CM: Role of inflammation in the pathogenesis of osteoarthritis: Latest findings and interpretations. Ther Adv Musculoskelet Dis 5: 77-94, 2013.

4. Swagerty DL Jr and Hellinger D: Radiographic assessment of osteoarthritis. Am Fam Physician 64: 279-286, 2001.

5. Kohn MD, Sassoon AA and Fernando ND: Classifications in Brief: Kellgren-Lawrence classification of osteoarthritis. Clin Orthop Relat Res 474: 1886-1893, 2016.

6. Hurley M, Dickson K, Hallett R, Grant R, Hauari H, Walsh N, Stansfield C and Oliver S: Exercise interventions and patient beliefs for people with hip, knee or hip and knee osteoarthritis: A mixed methods review. Cochrane Database Syst Rev 4: CD010842, 2018.

7. Bultink IE and Lems WF: Osteoarthritis and osteoporosis: What is the overlap? Curr Rheumatol Rep 15: 328, 2013.

8. Pouresmaeili F, Kamalidehghan B, Kamarehei M and Goh YM: A comprehensive overview on osteoporosis and its risk factors. Ther Clin Risk Manag 14: 2029-2049, 2018.

9. Sözen T, Özışık L and Başaran NC: An overview and management of osteoporosis. Eur J Rheumatol 4: 46-56, 2017.

10. Nazrun AS, Tzar MN, Mokhtar SA and Mohamed IN: A systematic review of the outcomes of osteoporotic fracture patients after hospital discharge: Morbidity, subsequent fractures, and mortality. Ther Clin Risk Manag 10: 937-948, 2014.

11. Dhital R, Lynn T, Tachamo N and Poudel DR: The trend of osteoporosis and osteoporotic fragility fractures in inpatients: Results from a national database. J Community Hosp Intern Med Perspect 9: 211-214, 2019.

12. Loeser RF: The role of aging in the development of osteoarthritis. Trans Am Clin Climatol Assoc 128: 44-54, 2017.

13. Palo N, Chandel SS, Dash SK, Arora G, Kumar M and Biswal MR: Effects of osteoarthritis on quality of life in elderly population of Bhubaneswar, India: A prospective multicenter screening and therapeutic study of 2854 patients. Geriatr Orthop Surg Rehabil 6: 269-275, 2015.

14. Lorenzo P, Aspberg A, Saxne T and Önnerfjord P: Quantification of cartilage oligomeric matrix protein (COMP) and a COMP neoepitope in synovial fluid of patients with different joint disorders by novel automated assays. Osteoarthritis Cartilage 25: 1436-1442, 2017.
15. Fernández-Puente $\mathrm{P}$, Calamia V, González-Rodríguez L, Lourido L, Camacho-Encina M, Oreiro N, Ruiz-Romero C and Blanco FJ: Multiplexed mass spectrometry monitoring of biomarker candidates for osteoarthritis. J Proteomics 152: 216-225, 2017.

16. van der Meijden OA, Gaskill TR and Millett PJ: Glenohumeral joint preservation: A review of management options for young, active patients with osteoarthritis. Adv Orthop 2012: 160923, 2012.

17. Sergé A: The molecular architecture of cell adhesion: Dynamic remodeling revealed by videonanoscopy. Front Cell Dev Biol 4: 36, 2016.

18. Liu F, Wang X, Zhang X, Ren C and Xin J: Role of serum cartilage oligomeric matrix protein (COMP) in the diagnosis of rheumatoid arthritis (RA): A case-control study. J Int Med Res 44: 940-949, 2016.

19. Samali A, Fulda S, Gorman AM, Hori O and Srinivasula SM: Cell stress and cell death. Int J Cell Biol 2010: 245803, 2010.

20. Green DR and Llambi F: Cell death signaling. Cold Spring Harb Perspect Biol 7: a006080, 2015.

21. Kim YL, Im YJ, Ha NC and Im DS: Albumin inhibits cytotoxic activity of lysophosphatidylcholine by direct binding. Prostaglandins Other Lipid Mediat 83: 130-138, 2007.

22. Kothari P, Johnson C, Sandone C, Iglesias PA and Robinson DN: How the mechanobiome drives cell behavior, viewed through the lens of control theory. J Cell Sci 132: jcs234476, 2019.

23. Mateos J, De la Fuente A, Lesende-Rodriguez I, FernándezPernas P, Arufe MC and Blanco FJ: Lamin A deregulation in human mesenchymal stem cells promotes an impairment in their chondrogenic potential and imbalance in their response to oxidative stress. Stem Cell Res (Amst) 11: 1137-1148, 2013.

24. Attur M, Ben-Artzi A, Yang Q, Al-Mussawir HE, Worman HJ, Palmer $G$ and Abramson SB: Perturbation of nuclear lamin A causes cell death in chondrocytes. Arthritis Rheum 64: 1940-1949, 2012.

25. Stephan JP, Roberts PE, Bald L, Lee J, Gu Q, Devaux B and Mather JP: Selective cloning of cell surface proteins involved in organ development: Epithelial glycoprotein is involved in normal epithelial differentiation. Endocrinology 140: 5841-5854, 1999.

26. Huang Z, Wang $X$ and Chen D: Signal proteins involved in myogenic stem cells differentiation. Curr Protein Pept Sci 18: 571-578, 2017.

27. Oh JE, Freilinger A, Gelpi E, Pollak A, Hengstschläger M and Lubec G: Proteins involved in neuronal differentiation of neuroblastoma cell line N1E-115. Electrophoresis 28: 2009-2017, 2007.

28. Grassel S and Aszodi A: Osteoarthritis and cartilage regeneration: Focus on pathophysiology and molecular mechanisms. Int J Mol Sci 20: 6156, 2019.

29. Akkiraju $\mathrm{H}$ and Nohe A: Role of chondrocytes in cartilage formation, progression of osteoarthritis and cartilage regeneration. J Dev Biol 3: 177-192, 2015.

30. Hornum L, Hansen AJ, Tornehave D, Fjording MS, Colmenero P, Wätjen IF, Søe Nielsen NH, Bliddal H and Bartels EM: C5a and C5aR are elevated in joints of rheumatoid and psoriatic arthritis patients, and $\mathrm{C} 5 \mathrm{aR}$ blockade attenuates leukocyte migration to synovial fluid. PLoS One 12: e0189017, 2017.

31. Xiang Y, Sekine T, Nakamura H, Imajoh-Ohmi S, Fukuda H, Nishioka K and Kato T: Proteomic surveillance of autoimmunity in osteoarthritis: Identification of triosephosphate isomerase as an autoantigen in patients with osteoarthritis. Arthritis Rheum 50: 1511-1521, 2004.

32. Takinami Y, Yoshimatsu S, Uchiumi T, Toyosaki-Maeda T, Morita A, Ishihara T, Yamane S, Fukuda I, Okamoto H, Numata $\mathrm{Y}$, et al: Identification of potential prognostic markers for knee osteoarthritis by serum proteomic analysis. Biomark Insights 8: 85-95, 2013.

33. Park YJ, Chung MK, Hwang D and Kim WU: Proteomics in Rheumatoid Arthritis Research. Immune Netw 15: 177-185, 2015.

34. Camafeita E, Lamas JR, Calvo E, López JA and FernándezGutiérrez B: Proteomics: New insights into rheumatic diseases. Proteomics Clin Appl 3: 226-241, 2009.

35. Mun S, Lee J, Lim MK, Lee YR, Ihm C, Lee SH and Kang HG: Development of a novel diagnostic biomarker set for rheumatoid arthritis using a proteomics approach. BioMed Res Int 2018: 7490723, 2018

36. Lee J, Mun S, Kim D, Lee YR, Sheen DH, Ihm C, Lee SH and Kang HG: Proteomics Analysis for Verification of Rheumatoid Arthritis Biomarker Candidates Using Multiple Reaction Monitoring. Proteomics Clin Appl 13: e1800011, 2019. 
37. Tan $\mathrm{K}$ and Lawler J: The interaction of Thrombospondins with extracellular matrix proteins. J Cell Commun Signal 3: 177-187, 2009.

38. Isenberg JS, Romeo MJ, Yu C, Yu CK, Nghiem K, Monsale J, RickME,WinkDA, FrazierWA and RobertsDD: Thrombospondin-1 stimulates platelet aggregation by blocking the antithrombotic activity of nitric oxide/cGMP signaling. Blood 111: 613-623, 2008.

39. Rouanne M, Adam J, Goubar A, Robin A, Ohana C, Louvet E, Cormier J, Mercier O, Dorfmüller P, Fattal S, et al: Osteopontin and thrombospondin-1 play opposite roles in promoting tumor aggressiveness of primary resected non-small cell lung cancer. BMC Cancer 16: 483, 2016.

40. Pinessi D, Ostano P, Borsotti P, Bello E, Guffanti F, Bizzaro F, Frapolli R, Bani MR, Chiorino G, Taraboletti G, et al: Expression of thrombospondin-1 by tumor cells in patient-derived ovarian carcinoma xenografts. Connect Tissue Res 56: 355-363, 2015.

41. Bender HR, Campbell GE, Aytoda P, Mathiesen AH and Duffy DM: Thrombospondin 1 (THBS1) promotes follicular angiogenesis, luteinization, and ovulation in primates. Front Endocrinol (Lausanne) 10: 727, 2019.

42. Ernens I, Bousquenaud M, Lenoir B, Devaux Y and Wagner DR: Adenosine stimulates angiogenesis by up-regulating production of thrombospondin-1 by macrophages. J Leukoc Biol 97: 9-18, 2015.

43. Tolsma SS, Volpert OV, Good DJ, Frazier WA, Polverini PJ and Bouck N: Peptides derived from two separate domains of the matrix protein thrombospondin-1 have anti-angiogenic activity. J Cell Biol 122: 497-511, 1993

44. Foulsham W, Dohlman TH, Mittal SK, Taketani Y, Singh RB, Masli S and Dana R: Thrombospondin-1 in ocular surface health and disease. Ocul Surf 17: 374-383, 2019.

45. Lee T,Esemuede N, Sumpio BE and Gahtan V: Thrombospondin-1 induces matrix metalloproteinase-2 activation in vascular smooth muscle cells. J Vasc Surg 38: 147-154, 2003.

46. Maumus M, Manferdini C, Toupet K, Chuchana P, Casteilla L, Gachet M, Jorgensen C,Lisignoli G and Noël D: Thrombospondin-1 partly mediates the cartilage protective effect of adipose-derived mesenchymal stem cells in osteoarthritis. Front Immunol 8: 1638, 2017.

47. McMorrow JP, Crean D, Gogarty M, Smyth A, Connolly M, Cummins E, Veale D, Fearon U, Tak PP, Fitzgerald O, et al: Tumor necrosis factor inhibition modulates thrombospondin-1 expression in human inflammatory joint disease through altered NR4A2 activity. Am J Pathol 183: 1243-1257, 2013.
48. Luo S, Li W, Wu W and Shi Q: Elevated expression of MMP8 and MMP9 contributes to diabetic osteoarthritis progression in a rat model. J Orthop Surg Res 16: 64, 2021.

49. Lioté F, Champy R, Moenner M, Boval-Boizard B and Badet J: Elevated angiogenin levels in synovial fluid from patients with inflammatory arthritis and secretion of angiogenin by cultured synovial fibroblasts. Clin Exp Immunol 132: 163-168, 2003

50. Iba K, Chiba H, Yamashita $T$, Ishii $S$ and Sawada $N$ : Phase-independent inhibition by retinoic acid of mineralization correlated with loss of tetranectin expression in a human osteoblastic cell line. Cell Struct Funct 26: 227-233, 2001.

51. Wewer UM, Ibaraki K, Schjørring P, Durkin ME, Young MF and Albrechtsen R: A potential role for tetranectin in mineralization during osteogenesis. J Cell Biol 127: 1767-1775, 1994.

52. Kamper EF, Kopeikina LT, Koutsoukos V and Stavridis J: Plasma tetranectin levels and disease activity in patients with rheumatoid arthritis. J Rheumatol 24: 262-268, 1997.

53. Kamper EF, Kopeikina LT, Trontzas P, Kyriazis NC, Vaiopoulos G and Stavridis J: Comparative study of tetranectin levels in serum and synovial fluid of patients with rheumatoid arthritis, seronegative spondylarthritis and osteoarthritis. Clin Rheumatol 17: 318-324, 1998.

54. Vincenti MP and Brinckerhoff CE: Early response genes induced in chondrocytes stimulated with the inflammatory cytokine interleukin-1beta. Arthritis Res 3: 381-388, 2001.

55. Sommaggio R, Pérez-Cruz M, Brokaw JL, Máñez R and Costa C: Inhibition of complement component $\mathrm{C} 5$ protects porcine chondrocytes from xenogeneic rejection. Osteoarthritis Cartilage 21: 1958-1967, 2013

56. Wang Q, Rozelle AL, Lepus CM, Scanzello CR, Song JJ, Larsen DM, Crish JF, Bebek G, Ritter SY, Lindstrom TM, et al: Identification of a central role for complement in osteoarthritis. Nat Med 17: 1674-1679, 2011

57. Silawal S, Triebel J, Bertsch $\mathrm{T}$ and Schulze-Tanzil G: Osteoarthritis and the complement cascade. Clin Med Insights Arthritis Musculoskelet Disord: Jan 3, 2018 (Epub ahead of print). doi: 10.1177/1179544117751430. 